\title{
Research on Real - time Image Processing Platform Based on Multi - core DSP
}

\author{
Xiaoming Liu \\ Department of Electrical and Information Engineering, Xi'an Jiaotong University City \\ College,Xi'an,710018,China
}

\begin{abstract}
In China, the information industry make progress of China's computer technology is also a step by step, about the exploration and application of image processing especially prominent, in particular, real-time image processing technology has been more attention. How to deal with massive data in real-time image processing is a worthy problem of our research in a limited time. In this paper, DSP as the core, pointing out the image processing aspects of the program and platform power supply, PCB design, and DSP - based real - time image processing platform for analysis of the advantages of the application.
\end{abstract}

Key words: multi - core, DSP, real - time image processing 、 platform Introduction

as the rapidly progress of image acquisition facilities, the high-definition image is to arrive to massive data processing, and a variety of complex computing algorithms to bring a huge amount of computation. Therefore, in some real-time requirements on the system more stringent circumstances, the image processing speed will affect the accuracy and effectiveness of the system ${ }^{[1]}$. In order to meet the requirements of fast and automatic image processing, this platform needs high-efficient data processing and high flexibility, so high-speed, high-energy multi-core DSP real-time image processing platform is to meet the fast processing image. Based on DSP chip TMS320C6201 as the core, according to real-time image processing, the image tracking as the background detection, real-time processing platform for in-depth study.

\section{1. real-time image processing platform works}

\subsection{Platform works}

Real-time image processing platform of the division of labor: real-time image processing platform includes four sections: DSP Module, image acquisition Module, the information output Module and logic control Module. In the specific application, usually use of CCD camera will capture the information collected to the image acquisition Module, through the D / A conversion, and then to the DSP Module, thus completing the image processing, and then output by the output Module processing results. How to deal with more and more complex images in a limited time is the main problem of real-time image processing platform. In accordance with the human visual 
effects, in order to ensure the real-time image, the platform and the image to be processed must be within 40 milliseconds.

1.2 Platform processing performance requirements

Image processing system has a strong theoretical and practical, it is mainly depends on the DSP technology. Image processing requirements of the more stringent requirements, many of which are above the GOPS. And because the use of different conditions, real-time image processing system to show the structure and functional characteristics are not the same. In short, real-time image processing platform to have the following characteristics ${ }^{[2]}$ :

(1) high computing performance: real-time image processing requirements on the platform that is the speed of computing requirements. Now the high-performance processing system to have a large number of sophisticated computing and data processing functions, it is also a key image processing system standard . It is mainly used in the complex case of multiple target tracking. In addition, massive real-time image processing, to have a platform, with high-speed data processing capabilities, data processing rate from MOPS to GOPS, but also to the future development of TOPS.

(2) real-time: the complexity of image processing platform is that in addition to the need for high computing performance, it must also meet the real-time needs. If used in military network communications, real-time testing and tracking of radar imaging, weapon navigation, etc., requires the processing system to complete the specified processing in a limited time. If you can not achieve real-time processing, it may lead to paralysis of the system.

(3) adaptability: image processing often face the image object and the image of the ever-changing back, as well as data fuzzy or noise and other aspects. With the detection of changes in the characteristics of the target will change, then there will be a different image processing methods. In a reasonable target recognition and tracking program, often have to choose the best algorithm to meet the target identification and tracking, which is the mixing image tracking thinking. Therefore, the need for image processing systems have a higher adaptability, used to complete various types of image processing ${ }^{[3]}$. Partial reconfiguration of the image processing system in motion is also one of the requirements of adaptability. This requires designers to note that the image processing system can be re-developed to meet the needs of the development of design staff to work in the platform to change the system structure or processing algorithms, but also to achieve the performance of speed and design flexibility needs. This flexibility requires avoiding the limitations of dedicated processors. In addition, in order to achieve the image processing process, incomplete image acquisition and variability, a variety of image algorithms from different aspects to find the characteristics of the target, to achieve image processing in the work of multi-mode parallel. The software designer can decompose the algorithm according to the task, make the processing result realize the highest parallel efficiency. At the same time, it is the key to the research of image processing to design the software and hardware of processing calculation method and establish a scientific and flexible hardware image processing system. 
(4) reliability: image processing platform often work in a bad environment. This requires real-time image processing platform has a certain reliability and stability, so as to adapt to such large temperature, corrosive, dusty external environment, so as to enhance the image processing system in the outdoor work ability, but also to increase image processing The useful life of the system.

2. Design of Multi - core DSP Real - time Image Processing Platform

For the multi-core DSP real-time image processing board is a real-time image processing platform of one part. A multi-core DSP real-time processing plate can also complete the image processing tasks alone, but also by a number of real-time image processing plate stitching into a real-time image processing platform, so many plates together to complete the image processing tasks. Designers choose multi-core DSP processor is also a lot of benefits ${ }^{[4]}$. First, the multi-core DSP processor can replace many separate DSP processors and many system controllers, but also highlights the advantages of its power. So to a large extent reduce the PCB area and overall consumption. Second, a number of DSP core information sharing between, to carry out seamless work, storage equipment, enhance the function, which also improves the overall speed. Third, the DSP core and external FPGA can use SRIO (serial fast IO) high-speed serial transmission of information exchange, rather than low-speed EMIFA connection. Fourth, multiple DSP core architectures can use many cores to maintain cache coherency, since a kernel can replicate its cache of up-to-date information directly into other cores, eliminating the need for additional memory.

2. 1. Multi-core DSP real-time image processing module function structure

DSP real-time image processing platform focuses on FPGA and three-core DSP components. There are many fiber optic transmission jacks and PCI Express jacks on the board, and FPGAs and multi-core DSP have a lot of storage space. DSP image processing hardware block diagram of the structure as shown in Figure 1.

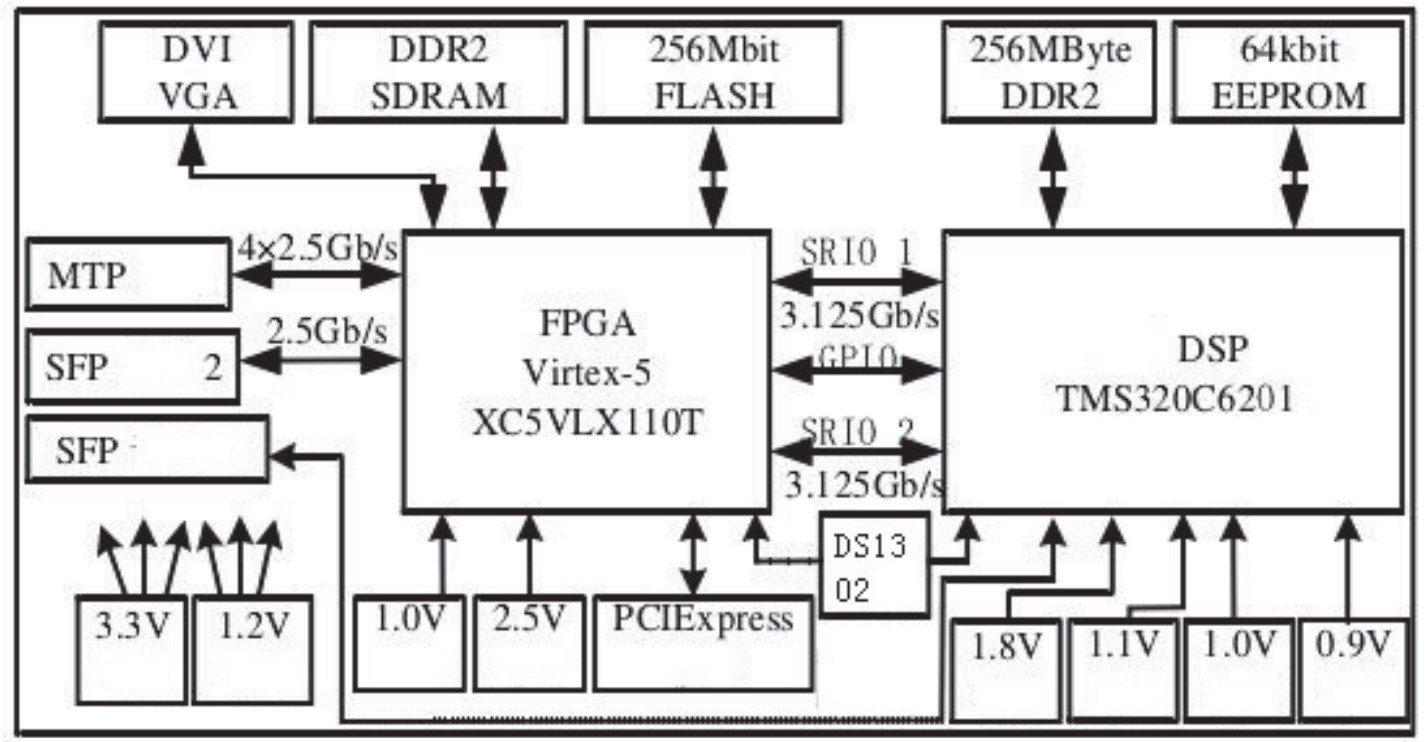

Figure 1 multi-core DSP real-time image processing module hardware block diagram of the structure

2.2 Image acquisition module

CCD camera is the image acquisition device, because the CCD derived 
analog data, so you can use the Philips SAA7111 to replace the DSP, to achieve image digitization and horizontal sync data isolation. Philips SAA7111 includes boundary scan test and I2C bus mastering chip registers. Philips SAA7111 chip operating frequency and the total chip into the crystal are $13.5 \mathrm{MHz}$ and 24.576 MHz. SAA7111 not only the role of the chip can be used to complete the storage device, but also allows users to have many choices.

\subsection{DSP module}

DSP module is the center of real-time image processing platform. Platform selection is TMS320C6201 chip, it needs CVdd and DVdd two power for supply voltage. When power is applied to both of these voltages, make sure that CVdd is first powered up. When turning off the power, make sure that DVdd is turned off first ${ }^{[5]}$. The platform uses a power supply and two voltage regulators to solve the power supply problem of CVdd and DVdd to meet the power supply demand of DSP. And the platform uses FIFO device between DSP board and image acquisition board. The digital image information is transmitted to FIFO through image acquisition board Device buffer, and then through the FIFO device, the image data to the DSP expansion of the SDRAM range, thus reducing the image transmission interrupt rate, improve the transmission speed.

Designers in accordance with the platform parallel processing scheme, the use of multiple instruction multiple data stream parallel architecture, composed of multiple processing blocks (including the algorithm processing equipment, hardware processing equipment, memory, etc.) at the same time work together to deal with various algorithms parallel structure. And in each image processing block, each plate has a corresponding processing channel. When the recognition features of the objects are extracted, they are self-processed according to the respective characteristics. Each image of the target and back separately, to find the corresponding characteristics, this link requires a lot of computing, so designers have special arrangements for real-time image processing section to deal with. All the image characteristics of the target summary, classification, integration and so complicated calculation, so the designer to the main control plate to deal with, so that the master processing section of information integration. And then according to the person in charge of the decision-making to deal with all the results of the plate integrated analysis, and finally select the best way to track control to achieve the goal of closely tracking. In many image parallel processing, the focus is on the division of tasks, but also all the image processing plate in all the results of the exchange, to achieve the goal of data integration. Therefore, the real-time image processing hardware architecture is an urgent need to address, memory, high-density real-time image processing module for each image data transfer between the integration of the problem.

\subsection{Output module and logic control module}

The output modules are divided into two types: single-chip RS232 module with RS232 jack and USB module with PDIUSBD12 socket. Two modules have some differences, RS232 module can only export the results of processing, USB module can not only export the results of the image, but also may monitor the DSP image data. DSP in the export to the use of TTL conversion, no longer need to use other 
conversion equipment, the output module and the logic control module is mutual influence in the image processing process, may have a FIFO and logic conversion problems, in order to solve this problem, Platform application CPLD equipment, so that a large extent to enhance the logic of the platform programmable functions.

\section{Platform Power and PCB Design}

3.1 Overall power consumption of the platform

Work in the platform, the system's stability and performance are closely related with the power, so when designing the power, be sure to very carefully. In this platform, play a key role in the device power consumption is relatively high, but also with multi-core DSP, so the actual design requirements and single-core processor also has a very big difference. Power dissipation in the platform to start, to TMS320C6021, XC5VLX 110 t, high-speed memory power analysis, to further draw the overall power consumption of the system.

\subsection{Platform power supply design}

In this platform, involves more power supply design, first of all to design the power of the overall program, and then in accordance with the overall requirements, followed by the design of each individual circuit, so you can ensure that the output power quality is relatively high, The stability of the power supply output can be ensured.

\subsection{Platform PCB design}

PCB design in the beginning before the first should be carefully analyzed, analysis, including cascading analysis, layout analysis and routing analysis, analysis of the signal after the need to expand the integrated design, so as to ensure the design of scientific. In addition, in the development of PCB design will face some problems, to find the corresponding solution.

\section{Analysis of the Advantages of the Application of Real - time Image Processing Platform Based on}

Because of the different needs of the market there have been a variety of image cards, but his versatility and handling are relatively poor, can not meet the system work needs. Real-time image processing platform includes: image acquisition, image processing, the level of interaction. Image processing is particularly troublesome, more data, the higher the relevance of the data, so the real-time image processing platform must have superior computing power. In real-time image work, if you want to enhance the system function, need to better highlight the image processing theory, such as: neural network in the use of image processing, boundary testing. As long as the platform into a versatile platform to use in different places in the original system work, can give full play to its versatility, such as: the platform can support a lot of image formats, you can choose a larger storage space.

\section{5. conclusion}

This article through the DSP real-time image processing platform to explore,make us clearly notice the real-time image processing platform practicality. In the actual use of the process that we gain a lot. Such as: computer vision technology, on-site analysis technology. With the gradual improvement of DSP technology, 
multi-core DSP technology in real-time image processing has been a wide range of use.

\section{references}

[1] SUN Ke-lin, ZHOU Wei-chao, WU Qin-zhang, et al Multi-core DSP image processing system for real-time optical fiber transmission $[\mathrm{J}]$ Optoelectronic Engineering, 2012, 39 (4): 136-144.

[2] Zhang Wenguang, Lu Min, Guo Yulan, et al Multi-core DSP laser imaging radar data processing system $[\mathrm{J}]$.

[3] SONG Dan.Image Real-time Correlation Matching Based on Multi-core DSP [J]. Communications Technology, 2015, 48 (4): 501-506.

[4] YE Yuan-guo.Design of High-speed Image Transmission and Processing System Based on FPGA and Multi-core DSP [J]. Electronic Testing, 2014 (9): 29-31.

[5] CAO Hai.Study on Laser Image Processing System Based on Pulse Coupled Neural Network [J] .Journal of Laser Technology, 2015 (10): 45-49. 